\title{
Image Retrieval by Utilizing Structural Connections within an Image
}

\author{
Pranoti P. Mane \\ Department of Electronics and Telecommunication, M.E.S. College of Engineering, SPPU, Pune, 411001, India \\ E-mail: ppranotimane@gmail.com
}

Narendra G. Bawane

S.B. Jain Institute of Technology, Management \& Research, Nagpur, 441501, India

E-mail: narendra.bawane@yahoo.com

\begin{abstract}
Content-based image retrieval (CBIR) is broadly applicable for searching digital images from a gigantic database. Images are retrieved by their primitive visual contents such as color, texture, shape, and spatial layout. The approach presented in this paper utilizes structural connections within an image by integrating textured color descriptors and structure descriptors to retrieve semantically significant images. The retrieval results were obtained by applying the HSV histogram, color coherence vector, and local binary pattern histogram to the standard database of Wang et al., which has 1000 images of 10 different semantic categories. Euclidean distance was used to find the similarity between the query image and database images. This method was evaluated against different methods based on edge histogram descriptors, color structure descriptors, color moments, the color histogram, the HSV histogram, Tamura features, edge descriptors, geometrical shape attributes, and statistical properties such as mean, variance, skewness, and kurtosis. Retrieval results obtained using the proposed methods demonstrated a significant improvement in the average precision $(73.8 \%$ and $73.1 \%$ ) compared with those obtained using other existing retrieval methods.
\end{abstract}

Index Terms - Content-based image retrieval, color coherence vector, local binary pattern, semantic gap, precision.

\section{INTRODUCTION}

Aiming to improve the efficiency of the image retrieval process, an image retrieval system proceeds toward a technique based on image contents known as contentbased image retrieval (CBIR).[1-7] In CBIR, the images are retrieved by their primitive visual contents such as color, texture, shape, and spatial layout. Multidimensional feature vectors, analogous to the visual contents of images, are extracted and stored in a feature database to build an image feature database. The example image is provided as a query by the user to retrieve relevant images. The system then converts these examples into its internal representations of feature vectors. The similarities between the feature vector of the query image and the database images are computed, and the retrieval is performed using an indexing scheme. This search is usually based on a similarity index rather than an exact match, and the retrieved results are then ranked according to the similarity index [1]. The general architecture of a CBIR system is shown in Fig. 1.

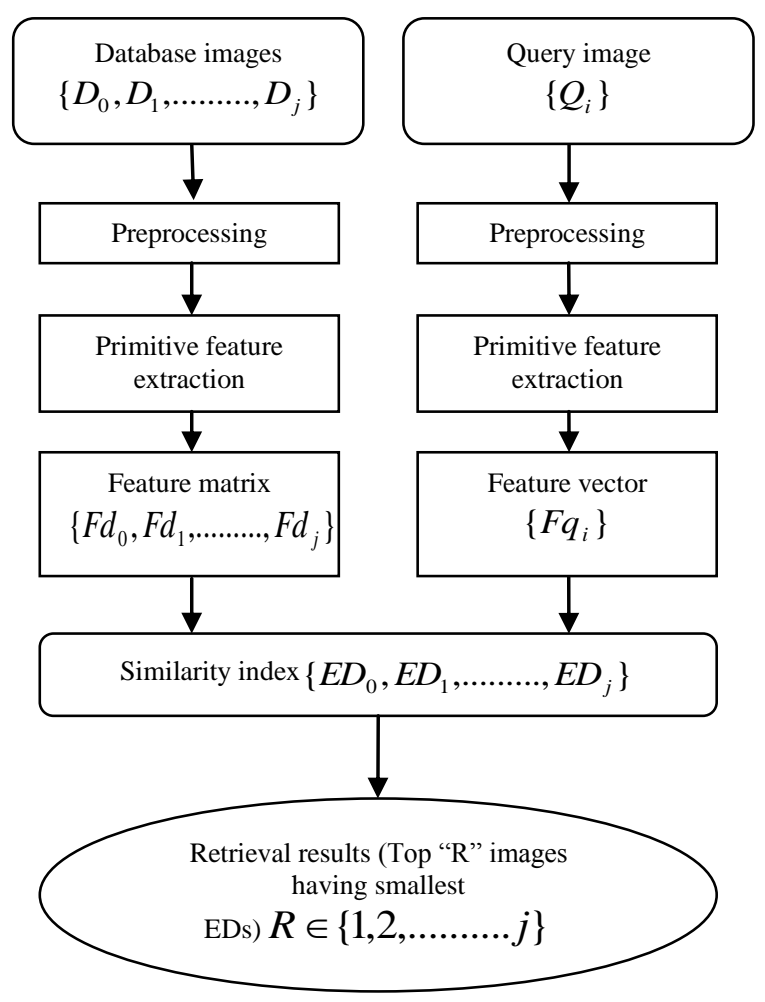

Fig.1. General Architecture of A CBIR System

The main concern in CBIR is the need for an effective and efficient feature extraction method for image representation, which conforms to the subjective human perception. This subjectivity transpires at all semantic levels while analyzing images because different users in the same situation or the same user in different circumstances may investigate or classify the same image differently. This inconsistency between image retrieval, by using low-level image features and high-level human semantics, is termed as the "semantic gap" [8-11].

In this study, we limited our goal to developing a 
general comprehensive algorithm for retrieving the images based on primitive visual features. Color is the most expansively used visual feature for image retrieval. Color features are relatively robust to the viewing angle, translation, and rotation of the regions of interest in an image. Although color is definitely not the most important visual quality of image data, it is a primitive image feature constituting a good launching point for developing a more sophisticated image retrieval system. In general, two types of image features are used to describe an image: (1) color features and (2) holistic structure features. The difference between these features is not always distinct. If spatial distribution is considered when extracting color features of an image, then the color features can be considered as holistic structure features. Thus, we used an approach to retrieve images based on color feature extraction by using two color descriptors: color histogram $(\mathrm{CH})$ and color coherence vector $(\mathrm{CCV})$. In addition, the local binary pattern (LBP), which amalgamates several orientations while keeping a low feature size, was used. The LBP transforms the relationship between two color levels into two levels (" 0 " or " 1 ") depending on a threshold represented by a binary code. This relationship of a pixel with eight neighbouring pixels is then transformed into a sequence of binary codes, resulting in an LBP level in the range of $0-255$.

The $\mathrm{CH}[12,13]$ represents the distribution of color contents effectively in an image when the color pattern is unique compared with the remaining the data set. The $\mathrm{CH}$ is easy and fast to compute and very robust for translation and rotation about the view axis. It is used for image retrieval by many commercial systems [14], such as QBIC, and academic systems such as NETRA, RETIN, KIWI, and Image Minor. Color moments (CMs) represent the color distribution of the images and are extensively used in QBIC, especially when an image comprises an object. Mostly, an image may not solely be described by its $\mathrm{CH}$ or the color distribution in it. One image may have a large number of scattered pixels with a particular color, whereas another may include the same amount of pixels with the same color but congregated in one area. Two images may have the same $\mathrm{CH}$, but a different spatial color arrangement. This information about the spatial location of colors in an image is represented by another color descriptor known as CCV [15]. The results of this study demonstrate that the retrieval efficiency $(73.8 \%)$ improves if we consider all the different aspects of an image by using the $\mathrm{CH}, \mathrm{CCV}$, and LBP.

The paper is organized as follows: Sec. 2 provides a rigorous literature survey. The methodology used in this paper is presented in Sec. 3. Other existing methods are mentioned briefly in this section as well. Sec. 3 also describes the proposed algorithm, whereas the experimentation is given in Sec. 4. The results of the proposed algorithm and their comparison with the existing methods are discussed in Sec. 5. Finally, Sec. 6 concludes the paper and throws light on the scope of future work.

\section{LITERATURE SURVEY}

Zhang et al. [16] extracted low-level features by using a 3-dimensional dominant color vector $(\mathrm{H}, \mathrm{S}$. and V) and a 24-dimensional Gabor feature vector. This paper proposed a new approach for digital image retrieval by using intermediate semantic features and multi-step search. This approach suggests a new direction from the existing image retrieval approaches, which works with high- or low-level semantic features. Unlike the existing systems in literature, the proposed system was capable of capturing regional and global features using semantic and low-level features. The results suggested that this system had notable advantages and is more promising compared with the existing techniques. In addition, it has a powerful SQL-based retrieval interface to support semantic and low-level retrieval.

$\mathrm{Li}$ et al. [17] represented the image by computing the HSV histogram as a color feature, pyramid wavelet transform (PWT) by using the Haar wavelet as a texture feature (in $\mathrm{YCbCr}$ color space), and an edge histogram as a shape feature (in $\mathrm{YCbCr}$ color space). This study focused on solving the small sample size problem and improving the capability of a kernel machine compared to traditional SVM-based RFs.

Hong et al. [18] used visual features, such as CM and wavelet moments, for computing feature vectors and comparing the query image and database images by using Mahalanobis distance.

Gosselin and Cord [19] exploited the color and texture information using the $\mathrm{L}^{*} \mathrm{a} * \mathrm{~b} *$ space and Gabor filters, respectively. Tests were conducted on the generalist COREL photo database containing 50,000 pictures. This method merged all the semantic information based on binary annotations provided by users during retrieval sessions. Therefore, the kernel matrix framework, which offers acceptable properties of matrices and efficient combinations with kernel-based techniques for image retrieval classifiers, was adopted.

Rui et al. [20] used the $\mathrm{CH}$ and CM and co-occurrence matrix for the COREL test set in an interactive approach using relevance feedback. Similarity matrix used for $\mathrm{CH}$ vectors was $\mathrm{CH}$ intersection and that for $\mathrm{CM}$ was Euclidean distance (ED). The authors also used the MESL test set for which the visual features used are the $\mathrm{CH}$, CM, Tamura, co-occurrence matrix, Fourier descriptors, and Chamfer shape descriptors. Chamfer matching was used for Chamfer shape representation, whereas weighted ED was used for the remaining features.

Broilo and Natale [21] presented the feature vector comprising of $32 \mathrm{CH}$ bins, $9 \mathrm{CMs}, 16$ edge histograms and wavelet texture energy values. The HVS color space was used to extract the CM, and the RB space was used for the $\mathrm{CH}$. Weighted $\mathrm{ED}$ was used for similarity matching. The image retrieval problem was formulated as an optimization problem and was solved by using particle swarm optimization.

Jiang et al. [22, 23] used a 9-dimensional CM in the LUV color space, a 64-dimensional CM in the HSV space, a 10-dimensional coarseness vector, and 8- 
dimensional directionality (Tamura's) texture features for low-level feature vectors. The experiments were conducted on COREL gallery. Generalized manifold ranking-based image retrieval for images was proposed in this study. The proposed method was observed to better than the existing learning methods such as MRBIR, whether or not the query image was in the database, which makes it more suitable for real applications.

Bian and Tao [24] used the $\mathrm{CH}$ in the HSV color space, a 128-dimensional CCV in the $\mathrm{L}^{*} \mathrm{a} * \mathrm{~b} *$ space, and a 9dimensional CM feature in the LUV color space. The texture and shape features were extracted using a wavelet transform and the edge directional histogram, respectively.

\section{Methodology AND PROPOSED AlgORITHM}

The primitive features required to compute an image feature vector are color and holistic structure features. If spatial distribution is considered when extracting color features of an image along with the LBP, then the resultant feature vector is considered to give high retrieval accuracy. Thus, we used an approach, based on feature extraction by integrating the $\mathrm{CH}$ in the HSV space, the $\mathrm{CCV}$, and the LBP histogram, for CBIR.

\section{A. Primitive Features}

In case of huge image data sets, $\mathrm{CHs}$ proved to be efficient and robust features for image indexing if the color pattern was unique compared with the remaining data sets. The $\mathrm{CH}[12,13,20]$ of an image portrays the frequency of each color level present in the image in the pixel domain. An image is quantized into sets of colors called bins to extract a $\mathrm{CH}$. The $\mathrm{CH}$ is then computed by counting the frequency or the number of times each quantized color level is present in the image. The $\mathrm{CH}$ is robust for translation and rotation, and tolerant to changes in the viewing angle, scale, and occlusion.

The CCV includes the information about the spatial location of colors in an image [15]. The CCV is constructed by first blurring the image and then quantizing the color space such that it only contains $n$ distinct colors. The $\mathrm{CH}$ of the image is then computed. Each histogram bin is then classified into two categories: coherent and incoherent. The pixel value is considered coherent when it belongs to a large, uniformly colored region, or it is considered to be incoherent. All the coherent and incoherent pixels of each bin are then counted and stored together in a vector. Thus, this forms a histogram-based CCV feature vector consisting counts of coherent and incoherent pixels of all the bins. The $\mathrm{CCV}$ corresponds to this classification for each color in the image. The dimensions of the $\mathrm{CCV}$ feature vector depend on the number of bins chosen when creating the histogram.

The LBP [25-27] is a simple method, which integrates different orientations by maintaining a small size of the feature vector. The LBP level varies from 0 to 255 and represents a spatial relationship between nine color levels in a pixel block of size $3 \times 3$ as shown in Fig. 2. The color level of the centre-most pixel is represented by $b$. For each block in the image, the center-most pixel level is compared with every neighbouring pixel value. When the color level of the neighbouring pixel is greater than or equal to the center-most pixel value, it is coded as 1 ; otherwise it is coded as 0 .

The LBP value of the block is calculated as shown in Equation (1).

$$
V_{L B P}=\sum_{x=0}^{7} f n\left(b_{x}-b\right) * 2^{x}
$$

where, the function " $f n$ " for the comparison is expressed as shown in Equation (2).

$$
f n(y)= \begin{cases}1 & y \geq 0 \\ 0 & y<0\end{cases}
$$

\begin{tabular}{|c|c|c|}
\hline $\mathrm{b}_{0}$ & $\mathrm{~b}_{1}$ & $\mathrm{~b}_{2}$ \\
\hline $\mathrm{b}_{7}$ & $\mathrm{~b}$ & $\mathrm{~b}_{3}$ \\
\hline $\mathrm{b}_{6}$ & $\mathrm{~b}_{5}$ & $\mathrm{~b}_{4}$ \\
\hline
\end{tabular}

Fig.2. Pixel Block of size $3 \times 3$.

The LBP levels fall between 0 and 255. The LBP histogram is computed as shown in Equation (3).

$$
\mathrm{H}_{\mathrm{LBP}}=\left\{h_{0}, h_{1}, \ldots \ldots, h_{255}\right\}
$$

Here $h_{i},(0 \leq i \leq 255)$ represents the frequency of an LBP level " $i$ " extracted from the complete image pixels.

\section{B. Proposed Algorithm}

- Load the database image $D_{n} D_{n} \in\left\{D_{i} \mid i=1,2, \ldots . .1000\right\}$

- Transform an RGB image into the HSV color space.

- Compute the feature vector $F \boldsymbol{i}=\left\{f_{h}, f_{l}, f_{c}\right\}$ where, $f_{h}$ is the HSV histogram, $f_{l}$ is the LBP histogram, and $f_{c}$ is the CCV of the database image.

- Compute the feature matrix $F_{D}$ of all database images. where, $F_{D}=\left\{F_{1}, F_{2}, \ldots \ldots \ldots, F_{1000}\right\}$

- Save $F_{D}$

- Input a query image $\mathrm{Q}_{\mathrm{i}}$

- Compute a feature vector $F_{q}=\left\{f_{h}, f_{l}, f_{c}\right\}$ where, $f_{h}$ is the HSV histogram, $f_{l}$ is the LBP histogram, and $f_{c}$ is the CCV of the query image.

- Calculate ED or the $\mathrm{L}_{2}$ metric as shown in Equation (4)

$$
E D(x, y)=\sqrt{\sum_{p=1}^{l}\left(x_{p}-y_{p}\right)^{2}}
$$


where, $\mathrm{x}_{\mathrm{p}}$ and $\mathrm{y}_{\mathrm{p}}$ are the 1-dimensional feature vectors of the database and query images, respectively.

- Compute ED for all the database images and retrieve the similar top 20 images having least ED.

We implemented the retrieval system using different feature extraction methods (Table 1) and compared the results to evaluate the performance of our proposed algorithm represented by proposed method 1 (PM-1) and proposed method 2 (PM-2).

Table 1. Details of the Methods Implemented (Numbers in Curly Braces Show Feature Length).

\begin{tabular}{|c|c|c|c|c|}
\hline $\begin{array}{l}\text { Name } \\
\text { of the } \\
\text { method }\end{array}$ & $\begin{array}{c}\text { Color } \\
\text { features }\end{array}$ & $\begin{array}{l}\text { Texture } \\
\text { features }\end{array}$ & $\begin{array}{c}\text { Shape } \\
\text { features }\end{array}$ & $\begin{array}{c}\text { Structure } \\
\text { features }\end{array}$ \\
\hline M-1 & $\begin{array}{c}\text { Color } \\
\text { structure } \\
\text { descriptors } \\
(\mathrm{CSD}) \\
\{128\}\end{array}$ & $\begin{array}{c}\text { Edge } \\
\text { histogram } \\
\text { descriptors } \\
\text { (EHD) } \\
\{150\}\end{array}$ & - & - \\
\hline M-2 & $\begin{array}{c}\mathrm{CH}\{9\} \\
\text { and } \\
\text { HSV } \\
\text { histogram } \\
\text { (HSV- } \\
\mathrm{H})\{9\}\end{array}$ & Tamura $\{4\}$ & Sobel $\{1\}$ & - \\
\hline M-3 & $\begin{array}{c}\mathrm{CH}\{24\} \\
\text { and } \\
(\mathrm{CM})\{7\}\end{array}$ & - & - & $\begin{array}{l}\mathrm{CCV} \\
\{48\}\end{array}$ \\
\hline M-4 & - & $\begin{array}{c}\text { Mean, } \\
\text { variance, } \\
\text { skewness, } \\
\text { and kurtosis } \\
\{20\}\end{array}$ & $\begin{array}{c}\text { Area, } \\
\text { eccentricity, } \\
\text { Euler number, } \\
\text { convex area, } \\
\text { perimeter, } \\
\text { orientation, } \\
\text { and Centroid } \\
\{40\}\end{array}$ & - \\
\hline PM-1 & $\begin{array}{l}\text { HSV- } \\
\mathrm{H}\{24\}\end{array}$ & $\operatorname{LBP}\{16\}$ & - & $\begin{array}{l}\text { CCV } \\
\{48\}\end{array}$ \\
\hline PM-2 & $\begin{array}{l}\mathrm{HSV}- \\
\mathrm{H}\{48\}\end{array}$ & $\operatorname{LBP}\{48\}$ & - & $\begin{array}{l}\mathrm{CCV} \\
\{48\}\end{array}$ \\
\hline
\end{tabular}

Image retrieval was performed using MPEG-7 color and texture descriptors [28-32]. The retrieval results of this method 1(M-1) are given in Table 2.

Furthermore, we integrated the shape feature along with the color and texture features [33] in method 2 (M2). The features used were Tamura (texture), RGB and HSV histogram (color), and Sobel operator (shape). "Hue histogram reduction" was used for a quick retrieval from a large database.

In this study, we implemented an algorithm for image retrieval using primitive color structure features $[15,20$, 22-24, 34]. Method 3, (M-3), used for image retrieval, was based on color features such as $\mathrm{CH}, \mathrm{CM}$, and color structure features such as CCV.

Another hybrid approach [11, 35] using shape and texture was used in method 4 (M-4). The shape features used were the area, eccentricity, Euler number, convex area, perimeter, orientation, and Centroid. The shape features are useful when an image has prominent objects. The first-order histogram-based features were used as texture features. Mean, variance, skewness, and kurtosis were used to detect the image texture. Similarity between features was measured using the ED.
We developed a new approach (PM-1 and PM-2) to improve the retrieval performance, which integrates the global basic color feature $(\mathrm{CH})$ and the features exploring spatial relationships (LBP and CCV). This approach amalgamates many orientations and maintains comparatively low feature size.

\section{EXPERIMENTATION}

Experiments were conducted using a standard database [36] of 1000 images. This database is a collection of colored images from 10 different categories such as African people, buildings, beaches, buses, dinosaurs, elephants, roses, horses, snowy mountains, and food plates. The image features were extracted using different primitive feature extraction methods, as shown in Table 1. In addition, similar features were extracted from the query and stored images. The distance between the query image and database images was computed, by calculating the ED or L-2 distance, and arranged in an ascending order array. The top 20 similar images were displayed and evaluated. The performance parameter i.e. precision is evaluated using Equation (5).

$$
\text { Precision }=\frac{R}{T}
$$

where, $\mathrm{R}$ is the number of relevant images retrieved and $\mathrm{T}$ is the total number of images retrieved.

\section{RESUlTS AND DISCUSSION}

The retrieval results based on MEG-7 descriptors are shown in Table 2. These results indicated that color structure features provide more information than texture features. However, it was not possible to retrieve all the semantic images through color features alone. Therefore, a combination of multiple features was adapted for the semantic image retrieval. It was observed that M-1 (the combination of color structure descriptor [CSD] and edge histogram descriptor [EHD] for feature extraction) proves to be useful for the retrieval of more semantically significant images.

The retrieval results vary according to the method used and the image type. For example, EHD and the integrated method show prominent results in the presence of a distinct object in the background, such as buses or flowers. CSD method is outperformed when an image comprises principal colors. The combined approach was found to be better for images with principal colors and distinct objects. Furthermore, the approach proved to work best when the database contained images, such as dinosaurs, which have similar texture, color, and background. The CSD and integrated method of color and texture work better in "multiple object images," which contain many objects such as horse, pony, fencing, and green field. However, the three approaches perform poorly when retrieving images from the food category. In spite of the fact that the average performance is more 
than $65 \%$, yet a feature vector length is huge (278). Along these lines computational time needed is high and it is cumbersome for a vast database.

Table 2. Retrieval results using MPEG-7 color-texture descriptors (M-1).

\begin{tabular}{|l|c|c|c|}
\hline \multicolumn{4}{|c|}{ Precision (\%) } \\
\hline $\begin{array}{l}\text { Image } \\
\text { category }\end{array}$ & $\begin{array}{c}\text { Feature } \\
\text { extraction } \\
\text { by CSD }\end{array}$ & $\begin{array}{c}\text { Feature } \\
\text { extraction by } \\
\text { EHD }\end{array}$ & $\begin{array}{c}\text { Feature } \\
\text { extraction by } \\
\text { CSD and EHD }\end{array}$ \\
\hline Building & 53 & 45 & 55 \\
\hline Bus & 73 & 96 & 97 \\
\hline Food plates & 51 & 36 & 45 \\
\hline Africans & 56 & 38 & 60 \\
\hline Beach & 39 & 32 & 48 \\
\hline Dinosaurs & 87 & 89 & 98 \\
\hline Elephants & 62 & 48 & 63 \\
\hline Flowers & 83 & 92 & 100 \\
\hline $\begin{array}{l}\text { Snowy } \\
\text { mountains }\end{array}$ & 46 & 30 & 47 \\
\hline Horses & 75 & 70 & 73 \\
\hline $\begin{array}{l}\text { Average } \\
\text { Performance }\end{array}$ & 62.5 & 57.6 & $\mathbf{6 8 . 6}$ \\
\hline
\end{tabular}

In another approach (M-2), we integrated texture features (Tamura) with color $(\mathrm{CH}$ and $\mathrm{HSV}-\mathrm{H})$ and shape (Sobel) features. Here only 23 features were used to reduce the computational time. Furthermore, the "hue histogram reduction" was used for quick retrieval from a large database [33]. Although three different primitive features were integrated in this method, no remarkable improvements were observed; the results were rather degraded in most cases (Table 3).

The method M-3 was used for image retrieval by using $\mathrm{CH}, \mathrm{CM}$, and $\mathrm{CCV}$. The feature vector length was moderate. The results were similar for most images. However, improvements were observed in the retrieval accuracy of images such as food plates and snowy mountains. Although the hybrid approach (M-4) used shape and texture features for feature extraction, it produced very poor results when compared to other feature extraction methods (Table 2).

Table 3. Category-wise results: average precision evaluated for the retrieval of five query images from each category.

\begin{tabular}{|l|l|l|l|l|l|l|}
\hline & \multicolumn{6}{|c|}{ Precision (\%) } \\
\hline \multirow{2}{*}{ Image category } & \multicolumn{7}{|c|}{ Methods implemented } \\
\cline { 2 - 8 } & M-1 & M-2 & M-3 & M-4 & PM-1 & PM-2 \\
\hline Building & 46 & 47 & 47 & 25 & 63 & 61 \\
\hline Bus & 96 & 83 & 87 & 17 & 93 & 95 \\
\hline Food plates & 45 & 44 & 53 & 21 & 70 & 61 \\
\hline African people & 54 & 49 & 55 & 23 & 68 & 72 \\
\hline Beach & 48 & 47 & 46 & 23 & 47 & 51 \\
\hline Dinosaurs & 98 & 99 & 98 & 92 & 100 & 100 \\
\hline Elephants & 66 & 59 & 58 & 41 & 64 & 52 \\
\hline Flowers & 99 & 56 & 80 & 37 & 88 & 89 \\
\hline $\begin{array}{l}\text { Snowy } \\
\text { mountains }\end{array}$ & 47 & 44 & 54 & 32 & 56 & 57 \\
\hline Horses & 75 & 98 & 85 & 21 & 89 & 93 \\
\hline $\begin{array}{l}\text { Average } \\
\text { Performance }\end{array}$ & 67.4 & 62.6 & 66.3 & 33.2 & $\mathbf{7 3 . 8}$ & $\mathbf{7 3 . 1}$ \\
\hline
\end{tabular}

Our approach (PM-1 and PM-2), which integrates the global basic color feature $(\mathrm{CH})$ and features exploring the spatial relationship (LBP and CCV), delivered improved results. This approach outperforms the existing methods of CBIR because of a significant improvement in the image retrieval of snowy mountains (56\%), buildings (63\%), African people (68\%), food plates $(70 \%)$, and dinosaurs (100\%). Our approach demonstrated comparable retrieval accuracy for other image categories as well. The retrieval results for a query image from the African category are shown in Fig. 3 and Fig.4. It is marked that along with good retrieval accuracy, the positive images were obtained at a lower rank, i.e. fast retrieval is achieved by using our proposed approach.
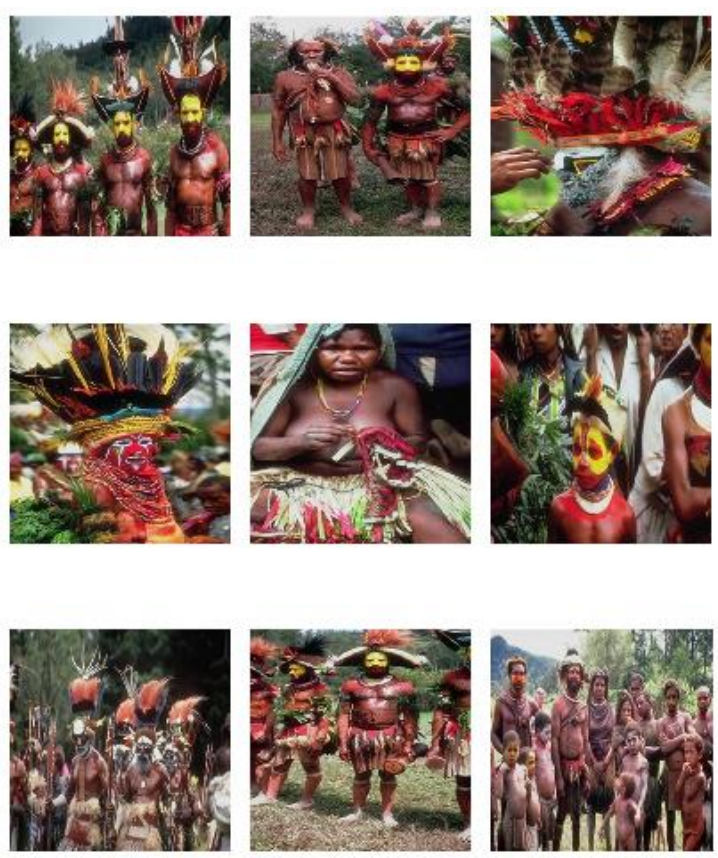

Fig.3. Retrieval Results for the Images of the 'Africans' by using the Proposed Method-1(Rank 1 to 9)
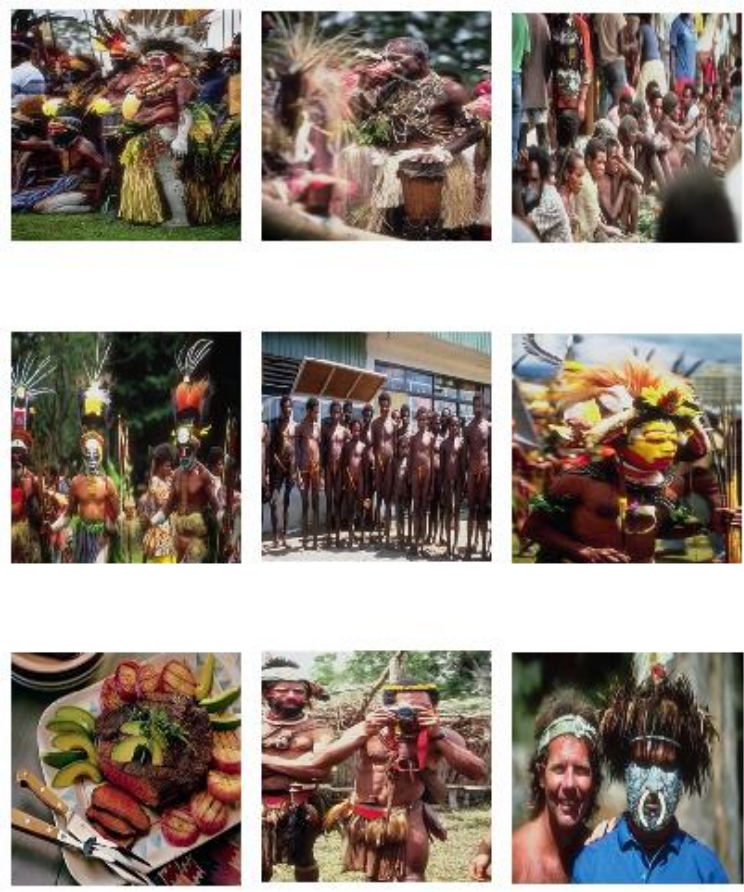

Fig.4. Retrieval Results for the Images of 'Africans' by using the Proposed Method-1(Rank 10 to 18) 
In the method PM-1, we have used 88 features which were less when contrasted with PM-2 (144 features). By this experimentation it is also observed that less computational time and better accuracy can be obtained by using optimum number of features as shown in Table 4.

Table 4. Effect of feature vector length on the speed and performance.

\begin{tabular}{|c|c|c|c|}
\hline \multicolumn{2}{|c|}{} & PM-1 & PM-2 \\
\hline \multirow{2}{*}{$\begin{array}{c}\text { Feature } \\
\text { vector } \\
\text { length }\end{array}$} & HSV histogram & 24 & 48 \\
\cline { 2 - 4 } & LBP histogram & 16 & 48 \\
\cline { 2 - 4 } & CCV & 48 & 48 \\
\hline \multicolumn{2}{|l|}{ Total feature vector length } & 88 & 144 \\
\hline \multicolumn{2}{|l|}{ Distance function } & ED & ED \\
\hline \multicolumn{2}{|l|}{ Computational time in seconds } & $\mathbf{7 5 8 . 4 9}$ & 907.90 \\
\hline \multicolumn{2}{|l}{ Average precision (\%) } & $\mathbf{7 3 . 8}$ & 73.1 \\
\hline
\end{tabular}

\section{CONCLUSION}

This study explored the feature extraction system that utilizes structural connections within an image by integrating textured color descriptors and structure descriptors to retrieve semantically significant images. Different feature extraction techniques were studied and implemented to evaluate and compare our proposed method, with the existing methods. After marking the limitations of the combination of other features, we introduced our integrated approach.

The main contribution of this study was the use of global color features and the features exploring the spatial relationship to amalgamate many orientations, textures, and color distributions among the images. Other improvements, such as minimizing the feature vector size and quickening the process, were proposed and discussed. Using a large number of bins was not necessary when extracting the histograms; rather it was observed that a better retrieval accuracy was obtained using 24 bins for HSV histogram and 16 bins for the LBP histogram. Our approach led to a fast and efficient CBIR system that is applicable to a variety of databases. Experiments using Wang's database showed that our method gives progressive results when compared with several other existing CBIR methods.

The CBIR system used in this paper may be extended to reduce the semantic gap in CBIR. The presented approach can be used for classifying image database to obtain more semantically meaningful retrieval results. The primitive features used in this paper may be mapped to high level semantic concepts by using relevance feedback algorithms.

\section{ACKNOWLEDGEMENT}

The authors would like to thank Dr. M. B. Kokare for his valuable discussions and guidance. The authors would also like to thank the anonymous reviewers for their valued suggestions in improving the quality of this paper.

\section{REFERENCES}

[1] M. B. Kokare, B. N. Chatterji and P. K. Biswas, "A
Survey on Current Content Based Image Retrieval Methods", IETE Journal of Research, pp. 261-271, 2002.

[2] Y. Liu, D. Zhang, G. Lu and W. Y. Ma, "A survey of content-based image retrieval with high-level semantics", Pattern Recogn. vol.40, issue1, pp. 262-282, January 2007.

[3] Pranoti Mane and Dr. N. G. Bawane, "Comparative Performance Evaluation of Edge Histogram Descriptors and Color Structure Descriptors in Content Based Image Retrieval", IJCA Proceedings on NCIPET 2013, No.6, pp. 5-9, December 2013.

[4] G. Rafiee, S. S. Dlay, and W. L. Woo, "A Review of Content-Based Image Retrieval”, CSSNDSP 2010, pp.775-779, 2010.

[5] Arnold W. M. Smeulders, Marcel Worring, Simone Santini, Amarnath Gupta,and Ramesh Jain, "Contentbased image retrieval at the end of the early years.", IEEE Trans. Pattern Anal. Mach. Intell., 22(12), pp.1349-1380, 2000.

[6] T. Tsai, Y. P. Huang and T. W. Chiang, "A fast two-stage content-based image retrieval approach in the DCT domain”, Intern. J. Pattern Recognit. Artif. Intell, vol.28, No. 4, pp. 765-781, 2008.

[7] Mohamed M. Fouad, "Content-based Search for Image Retrieval", IJIGSP, vol.5, no.11, pp.46-52, 2013.

[8] W.I. Grosky and R. Agrawal, "Narrowing the Semantic Gap in Image Retrieval: A Multimodal Approach", Multimedia Information Extraction and Digital Heritage Preservation, U. M. Munshi and B. B. Chaudhuri (Eds.), World Scientific, pp. 89-118.

[9] O. Karam, A. Hamad and M. Attia, "Exploring the semantic gap in CBIR: with application to lung CT", GVIP Conference (Cairo, Egypt), pp. 422-426, 2005.

[10] P. P. Mane, N. G. Bawane and A. Rathi, "Image retrieval using primitive feature extraction with hybrid approach", National Conference ACCET-15, India, pp. 99-102, 2015.

[11] A. J. M. Traina, J. Marques and C. Traina Jr, "Fighting the semantic gap on cbir systems through new relevance feedback techniques", Computer-Based Medical Systems, 19th IEEE International Symposium on, pp. 881-886, 2006.

[12] S.Maruthuperumal,G. Rosline Nesa Kumari, "A New Method for Content based Image Retrieval using Primitive Features", IJMECS, vol.5, no.10, pp.36-42, 2013.

[13] K. Prasanthi Jasmine, P. Rajesh Kumar,"Color Histogram and DBC Co-Occurrence Matrix for Content Based Image Retrieval", IJIEEB, vol.6, no.6, pp.47-54, 2014.

[14] M. Flickner, et al., "Query by image and video content: The QBIC system”, Computer, vol. 28, No.9, pp. 23-32, 1995.

[15] R. Zabih, J. Miller and G. Pass, "Comparing images using color coherence vectors", Proceedings of the fourth ACM international conference on Multimedia, pp. 65-73, 1996.

[16] D. Zhang, Y. Liu and J. Hou, "Digital image retrieval using intermediate semantic features and multistep search", DICTA, 513-518, 2008.

[17] J. Li, N. Allinson, D. Tao and X. Li, "Multitraining support vector machine for image retrieval", IEEE Trans. Image Process, vol.15, No.11, pp. 3597-3601, 2006.

[18] P. Hong, Q. Tian, and T. S. Huang, "Incorporate support vector machines to content-based image retrieval with relevant feedback", Image Processing, Proceedings, International Conference on., vol. 3, pp. 750-753, 2000.

[19] P. H. Gosselin and M. Cord, "Semantic kernel updating for content-based image retrieval", Multimedia Software Engineering, 2004. Proceedings, IEEE Sixth International Symposium on, pp. 537-544, 2004. 
[20] Y. Rui, T. S. Huang, M. Ortega and S. Mehrotra, "Relevance feedback: A power tool for interactive content-based image retrieval”, IEEE Trans. Circuits Syst. Video Technol, vol. 8, No.5, pp. 644-655, 1998.

[21] M. Broilo and F. G. B. De Natale, "A stochastic approach to image retrieval using relevance feedback and particle swarm optimization", Multimedia, IEEE transactions on, vol. 12, No. 4, 267-277, 2010.

[22] W. Jiang, G. Er, Q. Dai and J. Gu, "Similarity-based online feature selection in content-based image retrieval, IEEE Trans. Image Process, vol. 15, No.3, pp. 702-712, 2006.

[23] W. Liu, W. Jiang and S. F. Chang, "Relevance aggregation projections for image retrieval", Proceedings of the 2008 international conference on Content-based image and video retrieval, pp. 119-126, 2008.

[24] W. Bian and D. Tao, "Biased discriminant Euclidean embedding for content-based image retrieval", Image Process, IEEE Transactions on, vol. 19, No. 2, pp. 545$554,2010$.

[25] S. Joseph and K. Balakrishnan, "Multi-query content based image retrieval system using local binary patterns", International Journal of Computer Applications, vol. 17, No. 7, pp. 1-5, 2011.

[26] T. Mäenpää and M. Pietikäinen, "Texture analysis with local binary patterns", Handbook of Pattern Recognition and Computer Vision, vol. 3, pp. 197-216, 2005.

[27] V. Takala, T. Ahoven and M. Pietikainen, "Block-based methods for image retrieval using local binary patterns", Image Analysis, pp. 882-891, 2005.

[28] B. S. Manjunath, J. R. Ohm, V. V. Vasudevan and A. Yamada, "Color and texture descriptors", IEEE Trans. Circuits Syst. Video Techno, vol. 11, No. 6, pp. 703-715, 2001.

[29] Y J. R. Ohm, et al., "Color Descriptors, Introduction to MPEG-7: Multimedia Content Description Interface", eds. P. Salembier et al. (John Wiley \& Sons, New York, 2002), pp. 187-212.

[30] D. K. Park, Y. S. Jeon and C. S. Won, "Efficient use of local edge histogram descriptor", Proceedings of the 2000 ACM workshops on Multimedia, pp. 51-54, 2000.

[31] T. Sikora, "The MPEG-7 visual standards for content description-an overview", IEEE. Trans. Circuits. Syst. Video. Technol., vol. 11, No. 6, pp. 696-702, 2001.

[32] C. S. Won, D. K. Park and S. J. Park, "Efficient use of MPEG-7 edge histogram descriptor", ETRI Journal, vol. 24, No. 1, pp. 23-30, 2002.

[33] N. Singh, S. Dubey, P. Dixit and J. P. Gupta, "Semantic image retrieval by combining color, texture, and shape features", Computing Sciences (ICCS), IEEE
International Conference on. 2012, pp. 116-120, 2012.

[34] P. P. Mane and N. G. Bawane, "An approach to explore the role of color models and color descriptors in the optimization of semantic gap in content based image retrieval", International Journal of Computer Applications, vol. 104, No. 14, pp. 9-16, 2014.

[35] P. P. Mane and N. G. Bawane, Optimization of gap between visual features and high level human semantics in content based image retrieval, SCITECH, Pune, India, 2012.

[36] J. Z. Wang, James Z. Wang Research Group, Available at http://wang.ist.psu.edu.

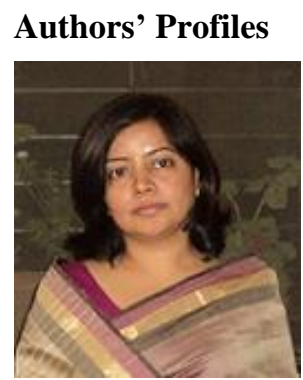

Pranoti P. Mane is currently pursuing her Ph.D. degree in the Department of Applied Electronics at S.G.B.A. University, Amravati, India. She received the B.E. degree from University of Aurangabad, India in 1998 and M.E. degree from S.G.B.A. University, Amravati, India, in 2006, all in Electronics Engineering. She has total teaching experience of more than 17 years. Her research interest includes image processing, signal processing, instrumentation and robotics.

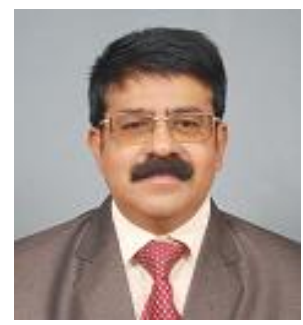

Narendra G. Bawane is currently working as a Principal, S.B. Jain Institute of Technology, Management and Research, Nagpur, India. He worked as a Head of the Computer Science and Engineering Department at G.H. Raisoni College of Engineering, Nagpur, India. He also worked with B.D. College of Engineering, Sewagram and Govt. Polytechnic, Nagpur, India. He has completed his B.E. from Nagpur University in 1987 and M. Tech. in 1992 from IIT, New Delhi. He completed his Ph.D. in 2006 at VNIT, Nagpur. He has total teaching experience of more than 25 years. His areas of interest include artificial neural network, wavelet analysis, image processing and emotion in speech and facial recognition, and hybrid intelligence.

How to cite this paper: Pranoti P. Mane, Narendra G. Bawane,"Image Retrieval by Utilizing Structural Connections within an Image", International Journal of Image, Graphics and Signal Processing(IJIGSP), Vol.8, No.1, pp.68-74, 2016.DOI: $10.5815 /$ ijigsp.2016.01.08 